\title{
CONSERVATION LAWS WITH DISCONTINUOUS FLUX FUNCTIONS
}

\author{
TORE GIMSE \\ Department of Mathematics \\ University of Oslo, Norway
}

\begin{abstract}
We study the initial value problem for the scalar conservation law $u_{t}+f(u)_{x}=0$ in one spatial dimension. The flow function may be discontinuous with a finite number of jump discontinuities. We prove existence of a weak solution, and the proof is constructive, suggesting a numerical method for the problem.
\end{abstract}

0. Introduction. In this paper we are interested in the Cauchy problem for the scalar conservation law:

$$
u_{t}+f(u)_{x}=0
$$

That is the initial value problem with $u(x, 0)=u_{0}(x)$ piecewise continuous of bounded variation, and so that $f_{0}(x)=f\left(u_{0}(x)\right)$ has bounded variation.

The flux function $f$ is supposed to be piecewise smooth with a finite number of jump discontinuities. For simplicity, we will consider flux functions with only one point of discontinuity, so that:

$$
\lim _{u \rightarrow \bar{u}^{-}} f(u) \neq \lim _{u \rightarrow \bar{u}^{+}} f(u),
$$

$\bar{u}$ being the point of discontinuity. The extension to a finite number of discontinuities is outlined at the end of the paper.

This Cauchy problem may arise in several physical applications. For two phase flow in porous media we may have a discontinuous flux (flow) function if the flow properties changes abruptly at some saturation. Such changes are obtained-for the relative permeability at the irreducible saturation, both when measuring the relative permeability experimentally [11],[16], and when modelling flow properties on a network of pores [12]. This effect is due to discontinuous distribution of the low saturation, and is a jump from zero permeability value to a presumably small but positive value at this critical saturation. Simulations on discretized fracture apertures indicate possible major discontinuities for the non-wetting phase relative permeability, particularly for systems with small longrange correlation among apertures in the direction of the flow [19]. A discontinuity of the

1980 Mathematics Subject Classification (1985 Revision). 35L65, 35L67, 76T05.

Key words and phrases. conservation laws, discontinuous flow, front tracking, porous media. Supported by the Royal Norwegian Council for Technical and Industrial Research 
relative permeability yields a corresponding jump for the flow function. In standard texts of reservoir simulation and related topics, e.g. [4], relative permeability curves are assumed to be continuous, or approximated by continuous functions. This paper however, suggests that also discontinuous functions, which in some cases may be more realistic, may be used with existence and stability results similar to those for the continuous problem.

It should be an object of further investigation if one could extend our results to be applicable also for hysteresis problems, that is, history dependent flow properties. Laboratory studies [6] indicate that one would expect to have an interval of saturations, say $\left(u_{1}, u_{2}\right)$ where $f(u)$ is double valued, and the correct flow value is determined by previous or neighboring saturation values. Marchesin et al. [17] have studied this problem, but their analysis is based on finite slopes of flow functions.

Another possible application is traffic flow analysis [15]. We propose the following model for two-lane unidirectional traffic on a freeway which involves a discontinuous flow function: Assume that all cars have the same length, and that the speed of cars in the left lane is constant, independent of the car density (at least at those values of interest here). In the right lane, a certain fraction of the cars drive with a low fixed speed, but passing (by changing lanes only during passing, and with instantaneous acceleration) is possible. Thus, as long as the density in the left lane permits passing, that is, as long as there is space enough between the cars, the overall flow depends continuously upon the overall density. However, as the density reaches the value where the left-lane density prohibits passing, the overall flow drops discontinuously to that of the two lanes considered separately. Although multilane traffic with passing has been studied previously (e.g. [18]), no model similar to the one proposed above is known by the author. The consequences of this model should be an object of future investigation.

In either application, the procedures of this paper are constructive, and suggest a numerical method. The major idea of our method is to approximate the flux function $f$ with a piecewise linear function, and approximate the initial value function $u_{0}$ with a step function [3], [7], [9]. By this procedure the original Cauchy problem is approximated by Riemann problems, and the solution of these consists of shocks only. We call this method a front tracking method. Shocks of the solution are traced without numerical dispersion, whereas rarefaction waves are approximated by a sequence of small shocks. Such methods have been extensively developed by the Oslo group [1], [2], and have turned out to be computationally and mathematically successful.

The following definition, simplifies the notation:

Definition. Let $u_{-}$and $u_{+}$denote the points $\left(\bar{u}, \lim _{u \rightarrow \bar{u}}-f(u)\right)$ and $\left(\bar{u}, \lim _{u \rightarrow \bar{u}}+f(u)\right)$ respectively. We write $u_{-} \lessdot u_{+}$if $\lim _{u \rightarrow \bar{u}^{-}} f(u)<\lim _{u \rightarrow \bar{u}}+f(u)$, and say that $f$ is double valued at the jump discontinuity at $u=\bar{u}$.

Throughout this paper we will assume that $u_{-} \lessdot u_{+}$. The case $u_{+} \lessdot u_{-}$can be treated symmetrically. We will treat $u_{-}$and $u_{+}$as being two different $u$ values, and we will let $\bar{u}$ denote any of them.

The fact that $f$ is discontinuous implies that the existence results of e.g. Krushkov [13] and Kuznetsov [14] do not apply to this problem. A somewhat similar "discontinuous problem" is the problem with a flux function discontinuously varying with $x$. This latter problem is solved in [5], by combining a technique of Temple [20] with front tracking 
methods by Dafermos [3] and Holden, Holden and Høegh-Krohn [9]. In this paper we will build mainly on [9]. By using front tracking as our method of analysis, we can avoid estimates involving the boundedness of the derivative of $f$, and thereby we are able to prove existence of a solution of the Cauchy problem. Our work will be based on, extend, and partly parallel, the previous works by Holden, Holden, and Høegh-Krohn [8], [9], where similar techniques are used to study the continuous case. As for their works, our method is based on the solution of Riemann problems for $(0-1)$, which will be discussed in some detail.

1. The solution of the Riemann problem. In general, the Riemann problem of $(0-1)$ is the initial value problem consisting of two constant states separated by a discontinuity,

$$
u(x, 0)= \begin{cases}u_{l}, & \text { for } x<0 \\ u_{r}, & \text { for } x>0\end{cases}
$$

The Riemann problem when neither of $u_{l}, u_{r}$ equals $\bar{u}$ is easily solved by the wellknown procedure of taking convex envelopes of $f$ between $u_{l}$ and $u_{r}$. Note that even though $f$ is not continuous between $u_{l}$ and $u_{r}$, the convex envelope of $f$ with respect to the interval $\left(u_{l}, u_{r}\right)$ is continuous and piecewise smooth. Thus, we obtain the familiar fan-like solution picture in the $x-t$ plane, of waves propagating with finite speed. In general the waves are smooth (rarefaction waves) or shocks. The latter being discontinuities traveling with a certain shock speed. A shock wave with left and right states $u_{1}$ and $u_{2}$ will be denoted a $u_{1} / u_{2}$ shock. However, since we may have for example $u_{l}<\bar{u}<u_{r}$, then, if $\bar{u}$ is part of the solution, one should specify whether one has $u_{-}$or $u_{+}$.

Special care should be taken when either $u_{l}$ or $u_{r}$ equals $\bar{u}$. The following lemma is easily verified by examining convex envelopes:

Lemma 1.1. The Riemann problem with initial values $u_{l}=\bar{u}$ and $u_{r} \neq \bar{u}$ has a unique solution with waves of finite speed only.

However, if $u_{r}=u_{-}<u_{l}$, or $u_{r}=u_{+}>u_{l}$, we have to extend the concept of convex envelopes:

Definition. The convex envelope of the function $f$ with respect to the interval $\left(u_{l}, u_{+}\right)$, where $f$ is double valued at $u_{-} \lessdot u_{+}$, is defined by the convex envelope of $f$ with respect to the interval $\left(u_{l}, u_{-}\right)$connected to the line from $u_{-}$to $u_{+}$.

The convex envelope defined above is a curve in the $u-f(u)$ space, which may have infinite slope with respect to $u$. Thus, a general Riemann problem (1-1) is solved by tracing the convex envelopes of $f$ with respect to the interval $\left(u_{l}, u_{r}\right)$, using the definition above if necessary. The solution generally consists of a fan of waves with finite speed, and possibly one shock $u_{-} / u_{+}$or $u_{+} / u_{-}$with infinite speed. Note that the Riemann problem $u_{l}=u_{-}$, $u_{r}=u_{+}$or vice versa, is solved by a single shock of infinite speed to the right in the $x-t$ plane. However, since the $u$ value is constant across such a shock, we call it a zero shock. Thus, in the sense of $u$, a zero shock carries no information, but the flux value information is transported instantaneously.

See Figure 1.1 for a simple example of a Riemann problem solution. 
2. Shock interactions. After the Riemann problem solution is found, we want to study the interaction of several Riemann problems. We will be particularly interested in the case of a piecewise linear flux function $f$, which implies that the only waves present are shocks [3],[9]. We define a single collision to be a collision involving and creating waves of finite speed only. That is, two or more waves interact at some point $(x, t)$, none of which has infinite speed, and the result contains no zero shock.

Starting out with finitely many Riemann problems as our initial data, we define the following algorithmic procedure for determining the solution $u(x, t)$ :

(1) Solve the initial Riemann problems, starting from the right along the $x$ axis. If a zero shock evolves, change the left state of the rightnext Riemann problem before solving that problem.

(2) After having finished at $t=0$, determine the first interaction to occur, say at $t=\tau$. Denote the interacting constant states by $u_{1}, u_{2}, \ldots, u_{M}, M>1$. Here $u_{1}$ is the leftmost state, and $u_{M}$ is the rightmost. The interaction is resolved by solving the Riemann problem with initial values $u_{l}=u_{1}$ and $u_{r}=u_{M}$. If a zero shock occurs, an interaction is created instantaneously at the rightnext front. If this happens, or if more interactions occur at the same time, treat them from the right, while changing the corresponding left value of the next front when zero shocks appear.

(3) When all interactions at $\tau$ are resolved, proceed to the next interaction at some greater time, etc.

As discussed above, a created zero shock, of course, will influence the rightnext front (or the rightnext interaction), but the following lemma assures limited distribution.

Lemma 2.1. A zero shock emerging from $(x, t)$ interacts with the rightnext front instantaneously, but only with the rightnext.

Proof. Assume that at $u_{-} / u_{+}$shock is formed. The rightnext front is necessarily of the kind $u_{+} / \tilde{u}$ where $\tilde{u} \neq \bar{u}$, since if $\tilde{u}=u_{+}$we had no front, and if $\tilde{u}=u_{-}$the rightnext front were a zero shock as well, which is impossible since our resolution starts from the right. Thus, the rightnext front is turned into a Riemann problem with initial states $u_{-}$and $\tilde{u}$, which, by Lemma 1.1, is solved by shocks of finite speed only. A similar argument is valid if the zero shock is a $u_{+} / u_{-}$shock.

If there is an interaction rightnext to the true collision, influence further to the right depends on the right state of that interaction.

Provided we have a finite number of interactions at time $t$, this completely resolves and continues the solution. It remains to be determined whether this procedure of resolution is well-defined, that is, whether the solution is independent of the order in which simultaneous interactions are resolved. Firstly, by Lemma 1.1 and 2.1, it is easily seen that the only cases that need to be checked are when more interactions occur with no fronts between them. The following lemma determines the resulting solution of a sequence of simultaneous interactions:

Lemma 2.2. For any finite sequence of simultaneous interactions creating zero shocks, the overall result is determined by the leftmost interaction. 
Proof. Let $I_{1}, I_{2}, \ldots I_{N}$ be the sequence of simultaneous interactions. Note that the left state of $I_{1}$ and the right state of $I_{N}$ may be different from $\bar{u}$, but that the rest of the left and right states involved equal $u_{-}$and $u_{+}$alternatingly. We will demonstrate that the order in which the interactions are treated does not affect the overall solution. Assume that the sequence of $I \mathrm{~s}$ is already obtained, and that the next interaction to consider is $I_{1}$. Assume that the right state of $I_{1}$ is $u_{-}$. The case of $u_{r}=u_{+}$is treated symmetrically. Thus, by the resolution of $I_{1}$, a $u_{+} / u_{-}$shock evolves, changing the left state of $I_{2}$ to $u_{+}$. However, by our assumption of the sequence, the right state of $I_{2}$ was $u_{+}$, so that the interaction $I_{2}$ is killed. The next interaction is not altered, and $I_{3}$ now is a new leftmost interaction in the remaining sequence. Thus, by continuing this argument, we see that the entire sequence is resolved by a $u_{+}$state, which was determined by the $u_{+} / u_{-}$shock emerging from the leftmost interaction.

Thus the resolution procedure defined by treating interactions with increasing $x$ is welldefined. We will define an event to be either a single collision, or one or more simultaneous interactions each creating zero shocks as described in Lemma 2.2. The latter will be denoted a dual collision. See Figure 2.1 for different kinds of events.

Having determined the well-defined algorithm for treating Riemann problems locally, we are now able to examine the procedure of solving a finite number of initial Riemann problems globally as $t \rightarrow \infty$. The following theorem extends a result from [9]:

Theorem 2.3. Given a piecewise linear flow function with one point of discontinuity, and an initial value function $u_{0}(x)$ consisting of finitely many constant states separated by discontinuities. Then, even for infinite time, only a finite number of events occur, and the overall solution $u(x, t)$ consists of a finite number of constant states, separated by shocks.

Proof. Let $N$ be the number of $u$ values between which $f$ is linear plus the number of initial $u$ values not in this set. Thus we may number the possible $u$ values $w_{1}, w_{2}, \ldots w_{N}$. Let $L(t)$ be the number of shock lines for $u(x, t)$, that is, the number of shock lines for a front $w_{i} / w_{j}$ is $|i-j|$, and let $F(t)$ be the number of shocks in $u(x, t)$. Define the function $G(t)=N L(t)+F(t)$. Then $G(t)$ is obviously non-negative. We will show that $G(t)$ is strightly decreasing at each event, leaving us with a finite number of possible events only. First, if the event is a true collision, the theorem from [9] is valid. Examine therefore a dual collision. We will compare the dual collision with two collisions, connected by a zero shock of large but finite speed (see Figure 2.2). Note that we may always find a speed $S$ so that no other interaction takes place before the shock with speed $S$ reaches the position of the right interaction. We name this the split case. Note that the result in the two cases are the same. Obviously, $G_{b e f o r e}$ and $G_{a f t e r}$ is the same for the two cases, and since we know that $G$ is decreasing for the split case [9], the same is valid for the dual collision. If more intermediate interactions were killed in between the left and right interaction, it is easily seen that $G$ decreases even more. Thus, we have a finite number of interactions, which gives only a finite number of shocks, dividing the $x-t$ plane in a finite number of polygons where the solution $u$ is constant.

Since the solution is piecewise constant, $G$ is proportional to the total variation. Thus: Corollary. The total variation of the solution is non-increasing. 
3. Stability. We now turn our interest to the stability of the solution, both with respect to $u_{0}(x)$, and the flux function $f(u)$. The following theorem ensures stability with respect to the initial data:

Theorem 3.1. If $u(x, t)$ and $v(x, t)$ solves (0-1) with initial value functions $u_{0}(x)$ and $v_{0}(x)$ respectively, $u_{0}$ and $v_{0}$ being step functions with finitely many values, and so that $u_{0}(x)=v_{0}(x)$ outside some finite interval $[-a, a]$, and $f$ being piecewise linear with one point of discontinuity, then

$$
\int|u(x, t)-v(x, t)| d x \leq \int\left|u_{0}(x)-v_{0}(x)\right| d x .
$$

Proof. Assume that $u_{0}(x)$ and $v_{0}(x)$ are constant at the intervals $I_{i}=\left(a_{i}, a_{i+1}\right)$, where $i=1,2, \ldots M$, and $a_{1}=-\infty, a_{M+1}=\infty$. We want to construct a sequence $\left\{u_{0, n}\right\}_{n=1}^{N}$ so that $u_{0,1}=u_{0}$ and $u_{0, N}=v_{0}$. This construction is done by taking the intervals $I_{i}$ one by one, and move the previous $u_{0, k}$ towards $v_{0}$ at one third of an interval every time. Thus, if $u_{0}=w_{s_{i}}$ and $v_{0}=w_{t_{i}}$ at interval $I_{i}$, then $N=\sum_{i=1}^{M} 3\left|s_{i}-t_{i}\right|$. Let $\left\{w_{j}\right\}$ be the set of initial and possible values for $u$. Note that $u_{0, i}$ differs from $u_{0, i+1}$ only at a third of some interval $I_{k}$, and that $\left|u_{0, i}-u_{0, i+1}\right|=\left|w_{j}-w_{j+1}\right|$ for some $j$ at this interval. Furthermore, $\left|u_{0}(x)-v_{0}(x)\right|_{L_{1}}=\sum_{i=1}^{N-1}\left|u_{0, i}-u_{0, i+1}\right| L_{1}$. Let $u_{i}(x, t)$ be the solution of $(0-1)$ with initial value $u_{0, i}$. We then have:

$$
\begin{gathered}
\int|u(x, t)-v(x, t)| d x \leq \int \sum_{i=1}^{N-1}\left|u_{i}-u_{i+1}\right| d x \leq \\
\int \sum_{i=1}^{N-1}\left|u_{0, i}-u_{0, i+1}\right| d x=\int\left|u_{0}(x)-v_{0}(x)\right| d x
\end{gathered}
$$

the latter inequality by Lemma 3.2 below, that is taken from [8]. $\square$

\section{Lemma 3.2 (Holden, Holden and Høegh-Krohn).}

$$
\int \sum_{i=1}^{N-1}\left|u_{i}-u_{i+1}\right| d x \leq \int \sum_{i=1}^{N-1}\left|u_{0, i}-u_{0, i+1}\right| d x
$$

Proof. The proof [8] considers the time derivative of $\int\left|u_{i}-u_{i+1}\right| d x$ at the intervals from Theorem 3.1. To transfer the result from [8], we observe that this derivative is zero also if $u_{i}=u_{-}$and $u_{i+1}=u_{+}$or vice versa.

Note that Theorem 3.1 implies stability also for higher dimensional problems. This follows by the dimensional splitting analysis by Holden and Risebro [10].

Next we are interested in stability with respect to the flux function $f$. At this point we will assume that the discontinuity of $f$ is fixed, and so are the two corresponding points $u_{-}$and $u_{+}$. With this assumption, we may state the theorem: 
Theorem 3.3. Let $f$ and $g$ be piecewise linear functions with a coinciding point of discontinuity at $u=\bar{u}$, and let $v(x, t)$ and $u(x, t)$ be the corresponding solutions of $u_{t}+f(u)_{x}=0$ and $v_{t}+g(v)_{x}=0$ with the same initial value, a step function taking finitely many values: $u_{0}(x)=v_{0}(x)$. Then

$$
\begin{array}{r}
\frac{d}{d t} \int|u(x, t)-v(x, t)| d x \leq T V_{x}\left(f\left(u_{c}(x, t)\right)-g\left(v_{c}(x, t)\right)\right) \\
\leq T V_{x}\left(f\left(u_{0, c}(x, t)\right)-g\left(v_{0, c}(x, t)\right)\right)
\end{array}
$$

where $u_{c}(x, t)$ and the Total Variation $\left(T V_{x}\right)$ are defined below.

Definition. Let $u_{i}$ be the value of the step function $u(x, t)$ taken at the interval $\left(a_{i}, a_{i+1}\right)$, $i=1,2 \ldots M$, for fixed $t$. Then $u_{c}(x, t)$ is defined by:

$$
u_{c}(x, t)= \begin{cases}u_{i}, & \text { for } a_{i} \leq x \leq a_{i+1}-\epsilon \\ u_{i}+\frac{\left(x-a_{i+1}+\epsilon\right)}{\epsilon}\left(u_{i+1}-u_{i}\right), & \text { for } a_{i+1}-\epsilon \leq x \leq a_{i+1} .\end{cases}
$$

Here $\epsilon=\frac{1}{3} \min _{i}\left\{a_{i+1}-a_{i}\right\}$.

Note that $u_{c}(x, t)$ is a piecewise linear, continuous function.

Definition. $T V_{x}(f(u(x)))$ is defined by

$$
T V_{x}(f(u(x)))=\sup \sum_{i=1}^{N}\left|f\left(u\left(x_{i+1}\right)\right)-f\left(u\left(x_{i}\right)\right)\right|
$$

where the supremum is taken over all finite partitions of $\left\{x_{i}\right\}$.

Note that $u$ in the above definition should be continuous.

Proof of Theorem 3.3. The proof of Theorem 3.3 carries over literally from [8] by the following observation. Define the function $F(u)=f(u)-g(u)$, and note that since $f$ and $g$ are assumed to have identical discontinuities, $F$ is continuous and piecewise linear. The analysis of [8] is based on estimates of $f-g$, and these estimates are still valid by the properties of $F$.

We now have stability results for piecewise linear flux functions with piecewise constant initial data, and we will use this, together with knowledge of zero shocks to conclude with existence and uniqueness results for problem $(0-1)$.

4. Existence and uniqueness. We first restate the problem that will be our object of study for the rest of this paper. The equation is:

$$
u_{t}+f(u)_{x}=0 \text {, }
$$

with initial data $u(x, 0)=u_{0}(x)$. The flux function $f$ is measurable and continuous with bounded derivative, except at $u=\bar{u}$ as above. The initial value function $u_{0}(x)$ is measurable and of bounded variation, as is $f_{0}(x)=f\left(u_{0}(x)\right)$. We assume there are values $u_{s}<\bar{u}<u_{S}$, and $x_{s}<x_{S}$, so that for $x \leq x_{s}$ and $x \geq x_{S}, u_{0}(x)$ is not in the interval $\left(u_{s}, u_{S}\right)$. The latter restriction is put on $u_{0}(x)$ to avoid zero shocks travelling unlimited distances instantaneously. We have the following lemma to ensure this: 
Lemma 4.1. There exist numbers $s$ and $S,-\infty<s<S<\infty$, and so that for $x<x_{s}+s t$ and $x>x_{S}+S t$ we have either $u(x, t)<u_{s}$, or $u(x, t)>u_{S}$. In these areas the solution $u(x, t)$ is determined by the existence and uniqueness results in [8].

Proof. Since $u_{0}(x)$ is of bounded variation, we may assume that $x_{S}$ is so that either $u_{0}(x)<u_{s}$ or $u_{0}(x)>u_{S}$ for $x>x_{S}$, and similarily for $x<x_{s}$. The maximum speed of waves entering the region $x>x_{\bar{S}}$ is then determined by the maximum slope of the function

$$
f_{S}(u)= \begin{cases}f(u), & \text { for } u \leq u_{-} \\ f\left(u_{-}\right)+\frac{f\left(u_{S}\right)-f\left(u_{-}\right)}{u_{S}-u_{-}}\left(u-u_{-}\right), & \text {for } u_{-} \leq u \leq \cdot u_{S} \\ f(u), & \text { for } u \geq u_{S} .\end{cases}
$$

By definition $f_{S}$ has a finite maximum slope, $S$. Similarily we define $f_{s}$ for waves entering the other region, $x<x_{s}$, and the lemma follows.

Before proceeding we need the following lemma from [8]:

Lemma 4.2. Assume that a measurable function $f$ is approximated by a sequence of measurable, uniformly bounded functions $\left\{g_{n}\right\}$ satisfying

$$
\left|g_{n}(x)-f(x)\right|<\frac{1}{n a_{n}}, \text { for } x \in(a, b)-A_{n}
$$

where the Lebesgue measure of $A_{n}, m\left(A_{n}\right)$ satisfies $m\left(A_{n}\right)<\frac{1}{n a_{n}},\left\{a_{n}\right\}$ being an increasing sequence of real numbers. Then for $m>n$, the sequence $\left\{g_{n}\right\}$ satisfies the following Cauchy criterion:

$$
\int_{a}^{b}\left|g_{n}(x)-g_{m}(x)\right| d x \leq \frac{2(b-a)}{n a_{n}}+\frac{4 M}{n a_{n}}
$$

where $M$ is such that $\left|g_{n}(x)\right|<M$.

We are now in the position of constructing a sequence of solutions, which we will show converges to a solution of (4-1): For given $k$, we select $k$ different $u$ values, say $w_{1}, w_{2}, \ldots, w_{k}$, among which we should have the two entries for $\bar{u}, u_{-}$and $u_{+}$. Then, for given $f$, we construct $f_{k}$ by evaluating $f$ at the chosen $u$ values, making $f_{k}$ piecewise linear between these values. Note that we by this construction keep the correct discontinuity. Finally we make a piecewise constant approximation of $u_{0}(x)$ from below, using only the $k$ different $u$ values at a finite number of sample points. We denote this approximation $u_{0, k}(x)$. Now, let $u_{k}(x, t)$ be the solution of the equation $u_{t}+f_{k}(u)_{x}=0$ with initial data $u_{0, k}(x)$. This defines a sequence of solutions, and we have the following lemma:

Lemma 4.3. $\left\{u_{i}(x, t)\right\}$ is a Cauchy sequence in $L_{1, l o c}$.

Proof. By the definitions made above, we apply Theorem 3.1,

$$
\int\left|u_{i}(x, t)-u_{j}(x, t)\right| d x \leq \int\left|u_{0, i}(x)-u_{0, j}(x)\right| d x+t T V_{x}\left(f_{i}\left(u_{0, i, c}(x)\right)-f_{j}\left(u_{0, j, c}(x)\right)\right) .
$$

As for the corresponding result in [8] the righthand terms vanish; the first by Lemma 4.2, and the second by the construction of $f_{i}$. Note that all $f_{i}$ have the same discontinuity at 
$\bar{u}$, and are continuous elsewhere. Thus, the function $F_{i j}$ defined by $F_{i j}(u)=f_{i}(u)-f_{j}(u)$ is continuous, which makes the second term vanish [8].

Since $f$ is double valued at $u=\bar{u}$, we cannot conclude from Lemma 4.3 that the sequence of fluxes, $\left\{f_{i}\left(u_{i}\right)\right\}$ converges. However, by the knowledge of the Riemann problem solution we find:

Lemma 4.4. If the original $u_{0}(x)$ is continuously increasing at $x_{0}$, where $u_{0}\left(x_{0}\right)=\bar{u}$, then for large $i$ the approximated solution contains $u_{-}$, and vice versa.

Proof. Since $u_{0}$ is continuously increasing, for $i$ sufficiently large, the approximation $u_{0, i}$ is also increasing at $x_{0}$. Thus, the Riemann problem solution of convex envelopes invokes $u_{-}$but not $u_{+} . \square$

Lemma 4.5. $\left\{f_{i}\left(u_{i}\right)\right\}$ is a Cauchy sequence in $L_{1, l o c}$.

Proof. By Lemma 4.3 we know that $\left\{f_{i}\left(u_{i}\right)\right\}$ is Cauchy with respect to domains where $\left\{u_{i}\right\}$ is not converging to $\bar{u}$. Thus, it is sufficient to examine initial values close to $\bar{u}$. This is a study of cases, of which the continuously monotone cases are covered by Lemma 4.4. The remaining are true Riemann problems, of which we may have only finitely many (by the restrictions of $u_{0}$ and $f_{0}$ ), and by the Riemann problem solution algorithm, we have convergence also for these.

We may now define the limiting functions of $\left\{u_{i}\right\}$ and $\left\{f_{i}\left(u_{i}\right)\right\}$ by defining the limit $u(x, t)$ to be the limit of $u_{i}(x, t)$ so that $f_{i}\left(u_{i}(x, t)\right) \rightarrow f(u(x, t))$. Note that this is a valid definition since by Lemma 4.3 we may define a family $\{\tilde{u}(x, t)\}$ so that for all $\tilde{u}$ in this family, $u_{i} \rightarrow \tilde{u}$ in $L_{1, l o c}$. The $\tilde{u}$ s differ only at sets of zero measure, or with respect to $u_{-} / u_{+}$. Thus, as $f$ is single valued, $f_{i}\left(u_{i}\right) \rightarrow f(u)$ in $L_{1, l o c}$, and the problem where $f$ is double valued is resolved by Lemma 4.5 , and thereby defining which $\bar{u}$ value to give the flux value $f(\bar{u})$.

Theorem 4.6. The limiting solution $u(x, t)$ defined above is a weak solution of the problem (4-1), that is:

$$
\int_{0}^{T} \int\left(u(x, t) \phi_{t}(x, t)+f(u(x, t)) \phi_{x}(x, t)\right) d x d t+\int u_{0}(x) \phi(x, 0) d x=0
$$

for all $\phi \in C_{0}^{1}$.

Proof. Since every $u_{i}(x, t)$ is a weak solution of $u_{t}+f_{i}(u)_{x}=0$, we have:

$$
\left|\int_{0}^{T} \int\left(u(x, t) \phi_{t}(x, t)+f(u(x, t)) \phi_{x}(x, t)\right) d x d t+\int u_{0}(x) \phi(x, 0) d x\right|=
$$




$$
\begin{aligned}
& \mid \int_{0}^{T} \int\left(\left[u(x, t)-u_{i}(x, t)\right] \phi_{t}(x, t)+\left[f(u(x, t))-f_{i}\left(u_{i}(x, t)\right)\right] \phi_{x}(x, t)\right) d x d t \\
& +\int\left(u_{0}(x)-u_{0, i}(x)\right) \phi(x, 0) d x \mid \\
& \leq \int_{0}^{T} \int\left(\left|u(x, t)-u_{i}(x, t)\right|\left|\phi_{t}(x, t)\right|+\left|f(u(x, t))-f_{i}\left(u_{i}(x, t)\right)\right|\left|\phi_{x}(x, t)\right|\right) d x d t \\
& +\int\left|u_{0}(x)-u_{0, i}(x)\right||\phi(x, 0)| d x .
\end{aligned}
$$

Now let $K=\max \left\{|\phi|,\left|\phi_{t}\right|,\left|\phi_{x}\right|\right\}$, and investigate each term of the above expression:

$$
\int_{0}^{T} \int\left|u(x, t)-u_{i}(x, t)\right|\left|\phi_{t}(x, t)\right| d x d t \leq K \int_{0}^{T} \int\left|u(x, t)-u_{i}(x, t)\right| d x d t \rightarrow 0
$$

and

$$
\int\left|u_{0}(x)-u_{0, i}(x)\right||\phi(x, 0)| d x \leq K \int\left|u_{0}(x)-u_{0, i}(x)\right| d x d t \rightarrow 0
$$

by the definition of $u(x, t)$ and $u_{0, i}(x)$. Finally, by Lemma 4.5 and the definition of $u(x, t)$ :

$$
\begin{aligned}
& \int_{0}^{T} \int\left|f(u(x, t))-f_{i}\left(u_{i}(x, t)\right) \| \phi_{x}(x, t)\right| d x d t \\
\leq K & \int_{0}^{T} \int\left|f(u(x, t))-f_{i}\left(u_{i}(x, t)\right)\right| d x d t \rightarrow 0 . \square
\end{aligned}
$$

Having proved existence of a weak solution, it remains to prove uniqueness of the solution. By uniqueness we mean that the constructive approach using front tracking gives a unique limit solution.

Theorem 4.7. The weak solution defined from Theorem 4.6 is the unique limit of the constructed sequence of piecewise constant solutions with respect to $L_{1, l o c}$.

Proof. Assume that both $v(x, t)$ and $u(x, t)$ are weak solutions of $(4-1)$ constructed by the front tracking method. Then

$$
\begin{aligned}
\Delta & =\int|u(x, t)-v(x, t)| d x \\
& \leq \int\left|u(x, t)-u_{i}(x, t)\right| d x+\int\left|u_{i}(x, t)-v(x, t)\right| d x \\
& \leq \int\left|u(x, t)-u_{i}(x, t)\right| d x+\int\left|u_{0, i}(x)-u_{0}(x)\right| d x+t \sum_{I} T V_{x}\left(f_{i}\left(u_{0, i, c}\right)-f\left(u_{0}\right)\right),
\end{aligned}
$$

the latter by Theorem 3.3 and $v_{0}(x)=u_{0}(x)$. The sum runs over intervals $I$ where $u_{0}$ is continuous. Thus, by the definitions of $u_{0, i}(x), u_{0, i, c}(x), u_{i}(x, t), u(x, t), f_{i}$, and $f, \Delta$ vanishes as $i \rightarrow \infty$. 
5. Finitely many discontinuities. The extension to a flow function with finitely many discontinuities where the one sided limits exist, are straightforeward by the observation that the zero shocks that may occur at each Riemann problem solution are well defined. By well defined, we mean that given $u_{l}$ and $u_{r}$, we may have only one zero shock traveling to the left, and one traveling to the right. By symmetry arguments, the results of this paper is valid for zero shocks traveling in both positive and negative direction. Zero shocks colliding at a dual collision are identical, and therefore the algorithmic procedure for solving multiple Riemann problems is still valid when being careful with changing the correct left and right states at neighboring fronts and interactions.

\section{REFERENCES}

1. F.Bratvedt, K.Bratvedt, C.F.Buchholz, T.Gimse, H.Holden, L.Holden, and N.H.Risebro, Front Tracking for Petroleum Reservoirs, Ideas and Methods in Mathematical Analysis, Stochastics, and Applications, Cambridge Univ. Press, 1992.

2. F.Bratvedt, K.Bratvedt, C.F.Buchholz, H.Holden, L.Holden, and N.H.Risebro, A New Front Tracking Method for Reservoir Simulation, SPE Res. Eng. (Feb.1992).

3. C.M.Dafermos, Polygonal Approximations of Solutions of the Initial Value Problem for a Conservation Law, J. Math. Anal. Appl. 38 (1972), 33-41.

4. R.E.Ewing (ed.), The Mathematics of Reservoir Simulation, Frontiers in Applied Mathematics, SIAM, 1983.

5. T.Gimse and N.H.Risebro, Solution of the Cauchy Problem for a Conservation Law with Discontinuous Flux Function, SIAM J. Math. Anal. (to appear).

6. R.E.Gladfelter and S.P.Gupta, Effect of Fractional Flow Hysteresis on Recovery of Tertiary Oil, SPE J. (Dec 1980), 508-520.

7. G.W.Hedstrom, Some Numerical Experiments with Dafermos Method for Nonlinear Hyperbolic Equations, Lecture Notes in Mathematics, Vol. 267, Springer-Verlag, Berlin-New York, 1972, pp. 117-138.

8. H.Holden and L.Holden, On Scalar Conservation Laws in One Dimension, Ideas and Methods in Mathematical Analysis, Stochastics, and Applications, Cambridge Univ. Press, 1992.

9. H.Holden, L.Holden, and R.Høegh Krohn, A Numerical Method for First Order Nonlinear Scalar Conservation Laws in One Dimension, Comput. Math. Applic. 15 (1988), 595-602.

10. H.Holden and N.H.Risebro, A Fractional Steps Method for Scalar Conservation Laws Without the CFL Condition, Preprint, Univ. of Oslo 15 (1991).

11. M.Honarpour and S.M.Mahmood, Relative-Permeability Measurements: An Overview, J. Pet. Tech. 40 (1988), 963-966.

12. A.Kantzas and I.Chatzis, Network Simulation of Relative Permeability Curves Using a Bond Correlated-Site Percolation Model of Pore Structure, Chem. Eng. Comm. 69 (1988), 191-214.

13. S.N.Krushkov, First Order Quasilinear Equations in Several Independent Variables, Math. USSR Sbornik 10 (1970), 217-243.

14. N.Kuznetsov, Weak Solutions of the Cauchy Problem for a Multi-Dimensional Quasilinear Equation, Mat. Zam. 2 (1967), 401-410.

15. M.J.Lighthill and G.B.Whitham, On Kinematic Waves. I. Flood Movement in Long Rivers, Proc. Roy. Soc. 229A (1955), 281-316; II. Theory of Traffic Flow on Long Crowded Roads, Proc. Roy. Soc. 229A (1955), 317-345.

16. B.B.Maini and T.Okazawa, Effects of Temperature on Heavy Oil-Water Relative Permeability of Sand, J. Can. Pet. Tech. 26 (1987), 33-41.

17. D.Marchesin, H.B.Medeiros, and P.J.Paes-Leme, A Model for Two Phase Flow with Hysteresis, Contemp. Math. 60 (1987), 89-107.

18. P.G.Michalopoulos, D.E.Beskos, and Y.Yamauchi, Multilane Traffic Flow Dynamics: Some Macroscopic Considerations, Transpn. Res.-B 18B (1984), 377-395.

19. K.Pruess and Y.W.Tsang, On Two-Phase Relative Permeability and Capillary Pressure of RoughWalled Rock Fractures, Water Resour. Res. 26 (1990), 1915-1926. 
20. B.Temple, Global Solution of the Cauchy Problem for a Class of 2x2 Non-strictly Hyperbolic Conservation Laws, Adv. Appl. Math. 3 (1982), 335-375.

Department of Mathematics, University of Oslo, P.O. Box 1053, Blindern, N-0316 Oslo 3 , NORWAY

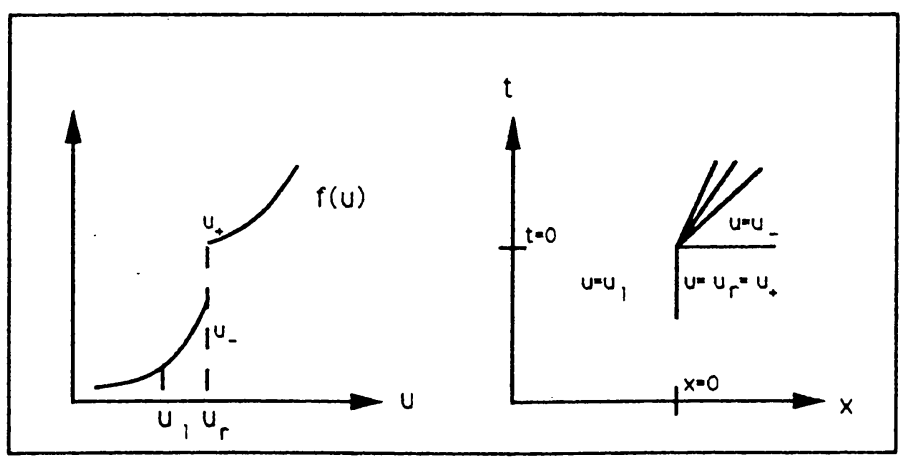

Figure 1.1 Discontinuous flux function and corresponding Riemann problem solution.

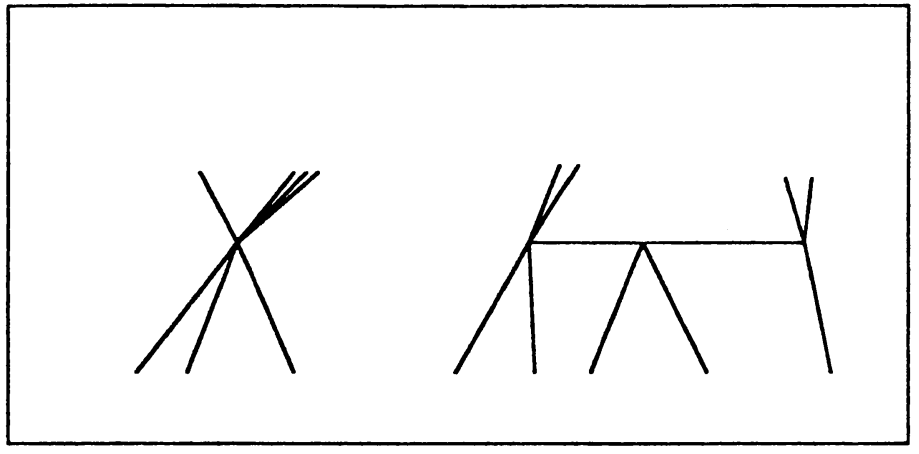

Figure 2.1 Single collision (left) and more interactions (right).

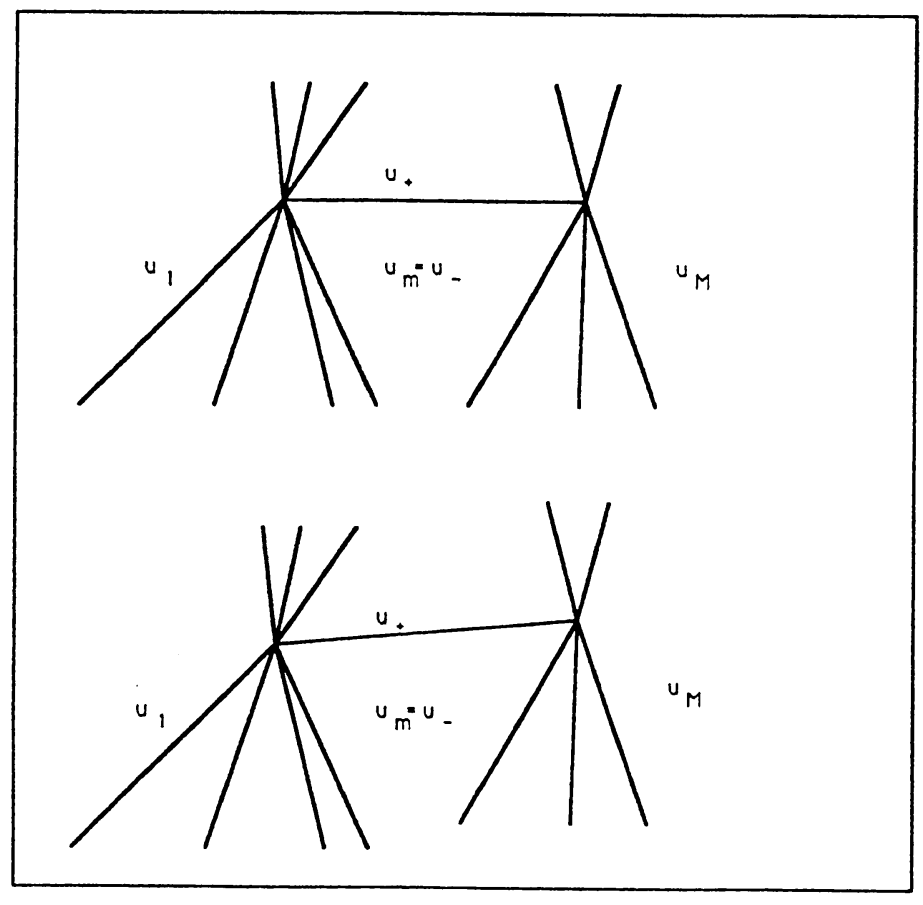

Figure 2.2 Original dual collision (top) and split collision (bottom) 